\title{
A New Surveillance Algorithm After Resection of Colorectal Liver Metastases Based on Changes in Recurrence Risk and RAS Mutation Status
}

\author{
Yoshikuni Kawaguchi, MD, PhD¹; Scott Kopetz, MD, PhD²; Heather A. Lillemoe, MD; Hyunsoo Hwang, PhD; \\ Xuemei Wang, MS'; Ching-Wei D. Tzeng, MD'; Yun Shin Chun, MD; \\ Thomas A. Aloia, MD, $\mathrm{MHCM}^{1}$; and Jean-Nicolas Vauthey, $\mathrm{MD}^{1}$
}

\begin{abstract}
Background: The optimal surveillance strategy after resection of colorectal liver metastases (CLM) is unknown. We evaluated changes in recurrence risk after CLM resection and developed a surveillance algorithm. Methods: Patients undergoing CLM resection during 1998 to 2015 were identified from a prospectively compiled database and analyzed if they had the potential for follow-up longer than the longest observed time to recurrence in this cohort. Changes in recurrence risk and risk factors for recurrence were evaluated. All statistical tests were 2-sided. Results: Among 2,105 patients who were initially identified and underwent CLM resection, the latest recurrence was observed at 87 months; 1,221 consecutive patients from 1998 through 2011 with the potential for at least 87 months of follow-up were included. The risk of recurrence was highest at 0 to 2 years after CLM resection, lower at 2 to 4 years after CLM resection, and steadily lower after 4 years after CLM resection. Factors associated with increased recurrence risk at the time of surgery were primary lymph node metastasis (hazard ratio $[\mathrm{HR}], 1.54 ; 95 \% \mathrm{Cl}$, 1.21-1.97; $P<.001)$, multiple CLM (HR, 1.31; 95\% Cl, 1.06-1.63; $P=.015)$, largest liver metastasis diameter $>5 \mathrm{~cm}(\mathrm{HR}, 1.64 ; 95 \% \mathrm{Cl}$, 1.23-2.19; $P<.001)$, and RAS mutation ( $\mathrm{HR}, 1.29 ; 95 \% \mathrm{Cl}, 1.04-1.59$; $P=.020$ ). In patients without recurrence at 2 years, the only factor still associated with increased recurrence risk was RAS mutation. In those patients, the recurrence rate at 4 years was $59.3 \%$ in patients with RAS mutation versus $27.8 \%$ in patients with RAS wild-type $(P=.019)$. Conclusions: For patients who have undergone CLM resection, we propose surveillance every 3 to 4 months during years 0 to 2 , every 3 to 4 months (if mutant RAS) versus every 4 to 6 months (if RAS wild-type) during years 2 to 4 , and every 6 to 12 months if recurrencefree at 4 years.
\end{abstract}

J Natl Compr Canc Netw 2020;18(11):1500-1508 doi: $10.6004 /$ jnccn.2020.7596

${ }^{1}$ Department of Surgical Oncology, ${ }^{2}$ Department of Gastrointestinal Medical Oncology, and ${ }^{3}$ Department of Biostatistics, The University of Texas MD Anderson Cancer Center, Houston, Texas.

\section{Background}

Approximately $15 \%$ of patients with colorectal cancer (CRC) have synchronous colorectal liver metastases (CLM; detected at initial diagnosis or during treatment after initial diagnosis), and approximately $30 \%$ of patients with CRC have metachronous CLM (detected after completion of treatment after initial diagnosis). ${ }^{1}$ Although surgical resection is the current standard of care for CLM, more than half of these patients experience recurrence. ${ }^{2-5}$ The putative purpose of surveillance after CLM resection is to detect recurrence early, when both the patient and the disease recurrence are treatable. Repeat surgery is effective for recurrence after CLM resection. Reported 5-year overall survival (OS) rates in patients who undergo resection of liver recurrence after initial CLM resection range from $41 \%$ to $73 \%,{ }^{6-10}$ and reported 5-year OS rates in patients who undergo resection of lung recurrence after CLM resection range from $39 \%$ to $54 \% .^{11,12}$

No study has evaluated the surveillance algorithm for patients undergoing resection of both synchronous and metachronous CLM. NCCN ${ }^{13,14}$ and the American Society of Colon and Rectal Surgeons (ASCRS) ${ }^{15}$ have published surveillance recommendations after surgery for stage IV CRC. ASCO has also published surveillance protocols for stage II and III CRC, but not protocols for surveillance after resection of metastatic disease due to the limited data to provide guidance. ${ }^{16}$ Given the paucity of recommendations for patients undergoing curative-intent resection of CLM, it is critical to explore this issue further and develop a potential surveillance algorithm specific to this patient cohort.

Within this context, the primary aim of this study was to assess the changes in recurrence risks and risk factors for recurrence over time in patients who underwent curative-intent resection of CLM.

See JNCCN.org for supplemental online content. 


\section{Methods}

\section{Patients}

A prospectively compiled database based on a dedicated institutional tumor registry was searched to identify patients who underwent liver resection for CLM from 1998 through 2015 at a tertiary academic center in Houston, Texas, that treats approximately 2,100 patients with CRC per year. We then determined the longest time to first recurrence after CLM resection among these patients. Next, to observe as many and as accurate numbers of recurrence after CLM resection as possible, we excluded patients who did not have the potential for a follow-up time after CLM resection that was at least as long as the longest time to first recurrence (in other words, patients for whom the interval between CLM resection and January 31, 2019, an end date for survival assessment, was shorter than the longest time to first recurrence). The study was approved by The University of Texas MD Anderson Cancer Center Institutional Review Board.

\section{Statistics}

Categorical variables were summarized using frequencies and percentages. Continuous variables were summarized using medians and interquartile ranges. Patients who were lost to follow-up or alive on January 31, 2019, were censored at the date of last known follow-up. Diagnosis of recurrence was based on the detection of a new lesion or a change in the size of a known lesion. Time to first recurrence and death was calculated from the time of CLM resection. Changing risks of recurrence and death were estimated using the kernel-smoothed hazard estimate method. ${ }^{17}$ OS was estimated using the Kaplan-Meier method. Cumulative incidence plots for disease recurrence were constructed and compared using the competing risk analysis proposed by Gray, ${ }^{18}$ in which death without recurrence was treated as a competing risk. A proportional hazards model was fit for recurrence-free survival. A backward elimination with a threshold $P$ value of .05 was used to select variables for the final models. Hazard ratios and 95\% confidence intervals were calculated for each factor. All statistical tests were 2 -sided, and $P \leq .05$ was considered to indicate statistical significance. Statistical analysis was conducted using SAS, version 9.4 (SAS Institute Inc).

\section{Results}

\section{Patients}

The database search revealed 2,105 patients who underwent initial CLM resection with curative intent from 1998 through 2015. Among these patients, the latest recurrence was observed 87 months after CLM resection. Thus, consecutive patients who underwent liver resection before October 2011 were included in the analysis, given that these patients had the potential for at least 87 months of follow-up (to the end date of January 31, 2019). A total of 1,221 patients from January 1998 through October 2011 were included in the analysis (supplemental eFigure 1, available with this article at JNCCN.org). Table 1 shows the demographic and clinicopathologic characteristics for all 1,221 patients and the 427 patients who had available genetic information regarding RAS mutation status. Prehepatectomy chemotherapy was delivered to 928 patients (76.0\%). Of the 1,221 patients, 786 (64.4\%) had died by the time of analysis, 851 (69.7\%) had experienced recurrence, and 98 (8.0\%) were censored without death before 87 months.

For patients who had experienced recurrence, reintervention (surgical resection, ablation, and/or radiation therapy) was performed in 391 (45.9\%) of the 851 patients with any type of recurrence after CLM resection, in 144 (64.6\%) of the 223 patients with liver recurrence alone, and in 106 (52.7\%) of the 201 patients with lung recurrence alone. Median duration of follow-up was 10.1 years (95\% CI, 9.7-10.4 years) based on the Kaplan-Meier method.

\section{Changing Risk of Recurrence and Death}

The risk of recurrence peaked approximately 1 year after CLM resection, diminished from 1 year to 4 years after resection, and remained fairly steady after 4 years after resection (Figure 1A). Recurrence risk was higher at 0 to 2 years after CLM resection (defined as the highrisk period) than at 2 to 4 years after CLM resection (intermediate-risk period), and remained low after 4 years (low-risk period). The risk of death peaked approximately 3 years after CLM resection and decreased slightly from 3 to 10 years after resection.

\section{Cumulative Recurrence and OS Rates Stratified by Time Free From Recurrence}

Given that the risk of recurrence was highest 0 to 2 years after CLM resection, intermediate 2 to 4 years after resection, and low after 4 years after resection, we evaluated the cumulative recurrence rate and OS rate for the entire cohort and then for patients who remained recurrence-free at 2 and 4 years. The 10-year cumulative recurrence rate was $72.8 \%$ for the entire cohort, $28.8 \%$ for patients without recurrence at 2 years, and $7.5 \%$ for those without recurrence at 4 years (Figure 1B). For the entire cohort, the cumulative recurrence rate at 2 years after CLM resection was $62.7 \%$. For patients who were recurrence-free at 2 years, the cumulative recurrence rate at 4 years after CLM resection was $23.2 \%$. For patients who were recurrencefree at 4 years, the cumulative recurrence rate at 6 years after resection was $5.1 \%$. The 10 -year OS rate was $33.2 \%$ for the entire cohort, $70.6 \%$ for the 2 -year recurrencefree group, and $87.2 \%$ for the 4 -year recurrence-free group (Figure 1C) 
Table 1. Demographic and Clinicopathologic Characteristics

\begin{tabular}{|c|c|c|}
\hline Characteristic & $\begin{array}{l}\text { All Patients } \\
(\mathrm{N}=1,221)\end{array}$ & $\begin{array}{l}\text { RAS Mutation Status Determined } \\
\qquad(\mathrm{n}=427)\end{array}$ \\
\hline \multicolumn{3}{|l|}{ Patient factors } \\
\hline Age, median (IQR) [range], y & $58(50-66)[19-88]$ & $55(48-63)[23-84]$ \\
\hline Sex, male:female, $n$ & 737:484 & $262: 165$ \\
\hline \multicolumn{3}{|l|}{ Race/Ethnicity, n (\%) } \\
\hline White & $983(80.5)$ & 341 (79.9) \\
\hline Hispanic or Latino & $136(11.1)$ & $43(10.1)$ \\
\hline Black/African American & $71(5.8)$ & $26(6.1)$ \\
\hline Asian American & $28(2.3)$ & $15(3.5)$ \\
\hline Other & $3(0.2)$ & $2(0.5)$ \\
\hline ASA score $\geq 3, n(\%)$ & $804(67.0)^{a}$ & $349(81.7)$ \\
\hline \multicolumn{3}{|l|}{ Primary lesion factors } \\
\hline Location, colon:rectum, $\mathrm{n}$ & $889: 332$ & $302: 125$ \\
\hline Right:Left & $325: 893^{a}$ & $105: 322^{a}$ \\
\hline T category $\geq 3, n(\%)$ & $1,055(88.1)^{\mathrm{a}}$ & $382(90.7)^{b}$ \\
\hline Lymph node metastasis, n (\%) & $776(65.5)^{a}$ & $308(73.9)^{\mathrm{b}}$ \\
\hline \multicolumn{3}{|l|}{ Liver metastasis clinical factors } \\
\hline Prehepatectomy CEA level, median (IQR), ng/mL & $3.4(1.6-13.5)$ & $3.0(1.4-9.4)$ \\
\hline CEA > 200 ng/mL, n (\%) & $40(3.3)$ & $6(1.4)$ \\
\hline Synchronous metastasis, n (\%) & $839(68.7)$ & $311(72.8)$ \\
\hline Extrahepatic disease, n (\%) & $61(5.0)$ & $39(9.1)$ \\
\hline Prehepatectomy chemotherapy, n (\%) & $928(76.0)$ & $374(87.6)$ \\
\hline With preoperative anti-VEGF agent, $n(\%)$ & $509(41.7)$ & $290(67.9)$ \\
\hline With preoperative anti-EGFR agent, $n(\%)$ & $68(5.6)$ & $45(10.5)$ \\
\hline \multicolumn{3}{|l|}{ Liver metastasis histopathologic factors } \\
\hline Tumor number, median (IQR) & $2(1-3)$ & $2(1-4)$ \\
\hline$\geq 2, \mathrm{n}(\%)$ & $674(55.2)$ & $268(62.8)$ \\
\hline Maximum tumor diameter, median (IQR), cm & $2.8(1.6-4.5)$ & $2.5(1.5-4.0)$ \\
\hline$>5, n(\%)$ & $222(18.2)$ & $62(14.5)$ \\
\hline R1 surgical margin, n (\%) & $110(9.0)$ & $58(13.6)$ \\
\hline RAS mutation, $\mathrm{n}(\%)$ & - & 179 (41.9) \\
\hline
\end{tabular}

Abbreviations: ASA, American Society of Anesthesiologists; EGFR, epidermal growth factor receptor; IQR, interquartile range; VEGF, vascular endothelial growth factor.

aData missing on ASA score for 21 patients, location (right vs left) for 3 patients, T category for 23 patients, and lymph node metastasis for 36 patients.

bData missing on T category for 6 patients and lymph node metastasis for 10 patients.

\section{Risk Factors for Recurrence}

A total of 416 patients had complete data on $R A S \mathrm{mu}-$ tation status, $\mathrm{T}$ category, and primary lymph node status. For these patients, a multivariable Cox proportional hazards model identified primary lymph node metastasis, multiple CLM, largest liver metastasis diameter $>5 \mathrm{~cm}$, and RAS mutation as risk factors for recurrence (Table 2). A Cox multivariable analysis that excluded RAS mutation status was also performed in the entire cohort and identified male sex, primary lymph node metastasis, multiple CLM, largest liver metastasis diameter $>5 \mathrm{~cm}$, extrahepatic disease, prehepatectomy chemotherapy, and R1 surgical margins as risk factors for recurrence (supplemental eTable 1).

Differences in Risk of Recurrence Over Time by Risk Factors for Recurrence

Analysis of recurrence risk over time by RAS mutation status showed that the risk of recurrence 0 to 4 years after CLM resection was higher for patients with $R A S$ mutation than for those with RAS wild-type (Figure 2A). Analysis of recurrence risk over time by number of CLM showed that the risk of recurrence 0 to 2 years after resection was higher for patients with multiple CLM than for those with 
A

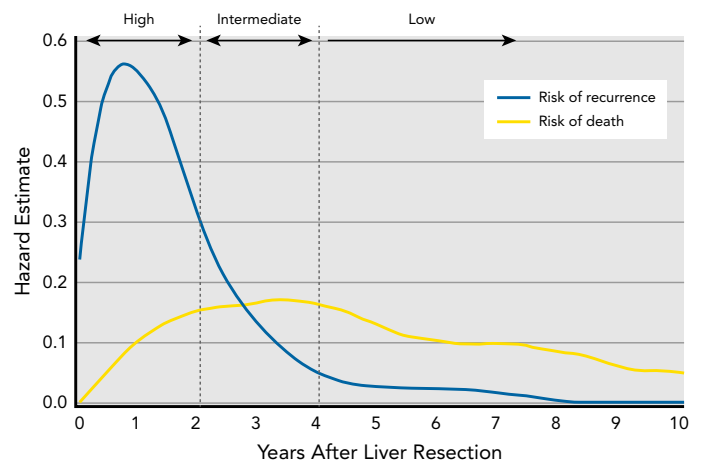

B
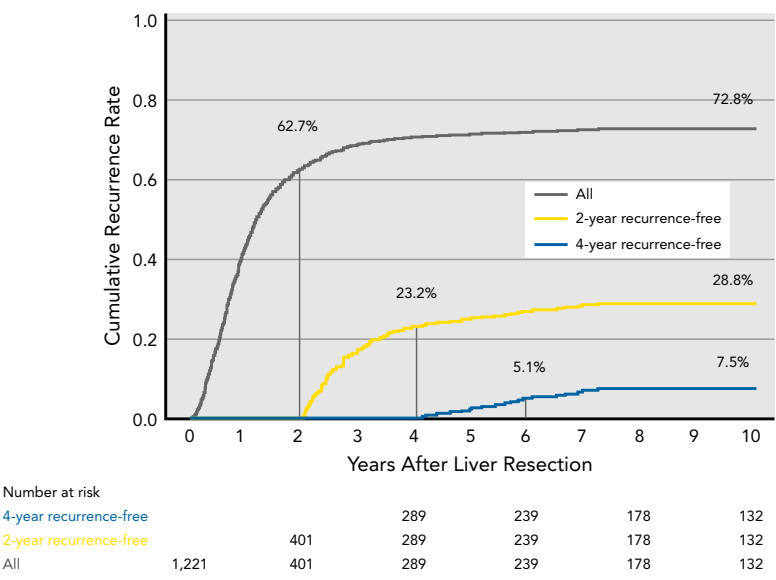

C

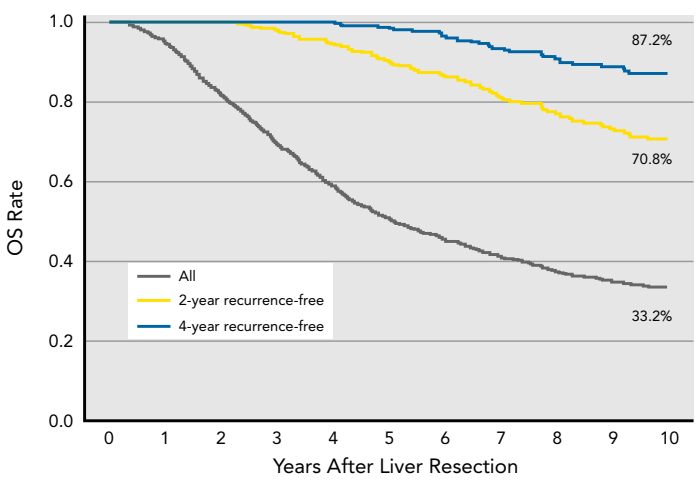

Number at risk

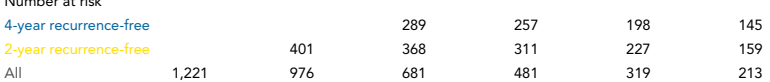

Figure 1. (A) Risks of recurrence and death over time after CLM resection. (B) Cumulative recurrence rate by competing risk analysis and $(C)$ OS rate in the entire cohort, 2-year recurrence-free group, and 4-year recurrence-free group.

Abbreviations: CLM, colorectal liver metastases; OS, overall survival.

single CLM, but the risk of recurrence after 2 years was similar for patients with multiple CLM and those with single CLM who were recurrence-free until that time (Figure 2B). When we compared patients according to primary lymph node status (Figure 2C) and largest liver metastasis diameter (data not shown), we found similar trends of increased risk of recurrence for patients with these risk factors 0 to 2 years after resection but not later.

Analysis of cumulative recurrence rates in patients who were free from recurrence at 2 years showed a significantly higher recurrence rate in patients with $R A S$ mutation than in those with $R A S$ wild-type $(P=.019$; Figure 2D). The recurrence rate at 4 years after resection was $59.3 \%$ in patients with $R A S$ mutation and $27.8 \%$ in those with $R A S$ wild-type. In contrast, in patients who were free from recurrence at 2 years, the cumulative recurrence rate was not significantly different between patients with multiple and single CLM $(40.9 \%$ and $35.2 \%$, respectively; $P=.500$; Figure $2 \mathrm{E}$ ) or between those with and without primary lymph node metastasis $(38.5 \%$ and $37.7 \%$, respectively; $P=.696$; Figure $2 \mathrm{~F}$ ). A multivariable Cox proportional hazards model identified RAS mutation as the only factor significantly associated with cumulative incidence of recurrence in patients free from recurrence at 2 years (hazard ratio, 2.29; 95\% CI, 1.23-4.26; $P=.009$ ) (supplemental eTable 2).

\section{Surveillance Recommendations}

The changes in risk of recurrence and risk factors for recurrence over time after CLM resection are summarized in Figure 3A. Our institution's proposed new surveillance strategy after CLM resection, based on the findings of this analysis, is as follows (Figure 3B): history and physical examination, CEA measurement, and axial imaging every 3 to 4 months during years 0 to 2 after CLM resection, every 3 to 4 months (if a patient has RAS mutation) or every 4 to 6 months (if a patient has $R A S$ wild-type) during years 2 to 4 , and every 6 to 12 months starting at 4 years. The postoperative surveillance strategy during years 2 to 4 is stratified by RAS mutation status because the risk of recurrence remains high during this period in patients with $R A S$ mutation who survive 2 years without recurrence. For comparison, surveillance recommendations of NCCN, ASCRS, and ASCO are summarized in Table 3.

\section{Discussion}

This analysis of changes in recurrence risk over time after CLM resection showed that the risk was highest during the initial 2 years after CLM resection, intermediate at 2 to 4 years after resection, and low after 4 years after resection. Although primary lymph node status, number of CLM, largest liver metastasis diameter, and RAS mutation were all risk factors for recurrence at the time of surgery, only $R A S$ mutation was significantly associated with recurrence in patients free from recurrence 2 years after resection. Based on these findings, for patients who have undergone CLM resection, we propose a surveillance 


\begin{tabular}{|c|c|c|c|c|c|}
\hline Variable & Patients, n & Events, $\mathbf{n}$ & Multivariable $\mathbf{H R}^{\mathbf{b}}$ & $95 \% \mathrm{Cl}$ & $P$ Value \\
\hline Yes & 308 & 269 & 1.54 & $1.21-1.97$ & $<.001$ \\
\hline No & 108 & 87 & Ref & & \\
\hline Multiple & 258 & 229 & 1.31 & $1.06-1.63$ & .015 \\
\hline Single & 158 & 127 & Ref & & \\
\hline \multicolumn{6}{|c|}{ Largest liver metastasis diameter, $\mathrm{cm}$} \\
\hline$>5$ & 61 & 57 & 1.64 & $1.23-2.19$ & $<.001$ \\
\hline$\leq 5$ & 355 & 299 & Ref & & \\
\hline
\end{tabular}

Abbreviations: CLM, colorectal liver metastases; HR, hazard ratio.

aBased on analysis of data from the 416 patients with complete data on RAS mutation status, T category, and lymph node metastasis.

${ }^{b}$ Cox proportional hazards model analysis initially included age ( $>60$ vs $\leq 60$ years), sex, primary tumor location, $T$ category, primary lymph node metastasis, prehepatectomy CEA level ( $>200.0$ vs $\leq 200.0 \mathrm{ng} / \mathrm{mL}$ ), synchronous metastases (vs metachronous metastases), prehepatectomy chemotherapy, extrahepatic disease, number of CLM (multiple vs single), largest liver metastasis diameter ( $>5 \mathrm{vs} \leq 5 \mathrm{~cm}$ ), surgical margin status (R1 vs R0), and RAS mutation status. A backward elimination with a threshold $P$ value of .05 was used to select variables for the final models.

algorithm integrating RAS mutation status. The surveillance protocol has the highest intensity during the initial 2 years after CLM resection, with a stepwise reduction in intensity with increased time after resection if recurrencefree. However, for patients with RAS mutation, we recommend continuing high-intensity surveillance beyond 2 years after CLM resection.

Our findings indicated that recurrence rates after resection of CLM were higher than previously reported recurrence rates after resection of primary colorectal tumors. Specifically, we found that the recurrence rate was $62.7 \%$ at 2 years after CLM resection, $23.2 \%$ at 4 years after resection in patients who were recurrence-free at 2 years, and $5.1 \%$ at 6 years after resection in patients who were recurrence-free at 4 years. In contrast, previous studies showed that the recurrence rate at 2 years after resection of primary CRC was approximately $5 \%$ to $25 \%^{19-21}$ and differed by stage and primary tumor site (colon or rectum). Specifically, Tsikitis et $\mathrm{al}^{19}$ found that the 2-year recurrence rate after resection of primary CRC was $6.0 \%$ for stage I-IIA disease and $23.7 \%$ for stage IIB-III disease, Wille-Jørgensen et $\mathrm{al}^{20}$ found that the 2-year recurrence rate after resection of stage II-III primary CRC was approximately $10.0 \%$ to $15.0 \%$, and Snyder et $\mathrm{al}^{21}$ found that the 2-year rate after resection of stage I-III CRC was approximately $10.0 \%$ to $20.0 \%$. Therefore, the recurrence rate at 2 years after resection of CLM is approximately 3 times the recurrence rate at 2 years after resection of primary colorectal tumors, but the recurrence rate at 4 years after CLM resection in patients who are recurrence-free at 2 years is similar to the recurrence rate at 2 years after resection of primary colorectal tumors.

Another clinically relevant finding of this study was that the duration of the risk effect was longer for the molecular biomarker that we examined, RAS mutation status, than for the traditional clinicopathologic factors, including liver metastasis factors (number of CLM and largest liver metastasis diameter) and primary lesion factor (lymph node status). In light of this finding, we suggest continued high intensity of surveillance beyond 2 years after CLM resection for patients with RAS mutation.

Taken together, our study is also helpful for counseling a patient's prognosis after a given recurrence-free time interval while accounting for $R A S$ mutation status.

The putative purpose of surveillance strategies to detect asymptomatic and treatable recurrence has been described in patients with resected primary colorectal tumors, ${ }^{22,23}$ breast cancer, ${ }^{24}$ and pancreatic adenocarcinoma. ${ }^{25,26}$ However, a protocol for surveillance after CLM resection that covers both synchronous and metachronous CLM has not yet been established. Given that patients able to undergo repeat liver resection ${ }^{6-10}$ or lung resection ${ }^{11,12}$ at the time of recurrence after CLM resection have favorable survival rates compared with patients undergoing palliative nonoperative therapy, a surveillance protocol matched with the risk of recurrence after CLM resection may be helpful for detecting early manifestation of recurrence and may facilitate repeat intervention. Our surveillance algorithm requires more frequent testing and clinic visits for patients within the 0 to 2 years after CLM resection compared with other algorithms; however, given 
A

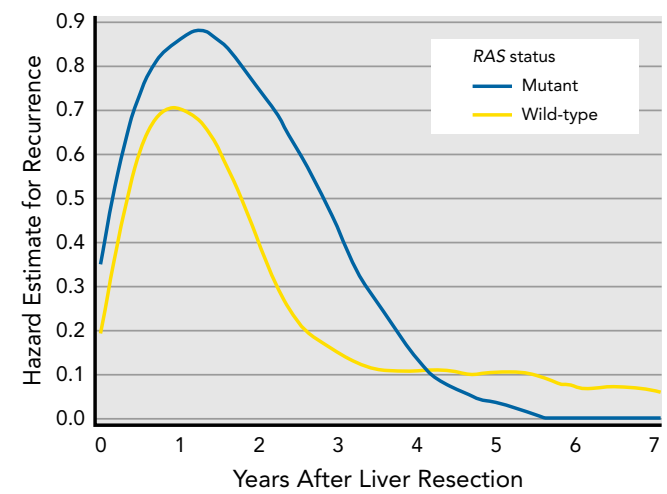

B

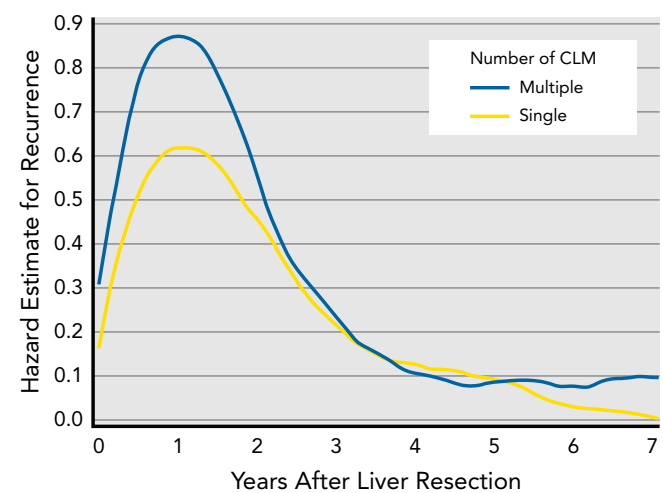

C

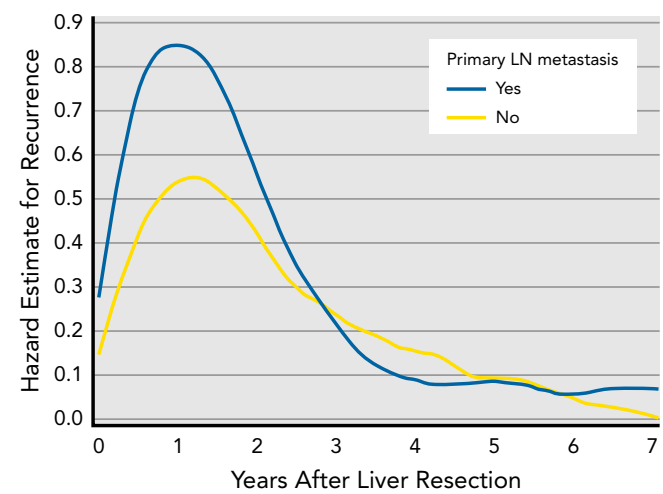

D

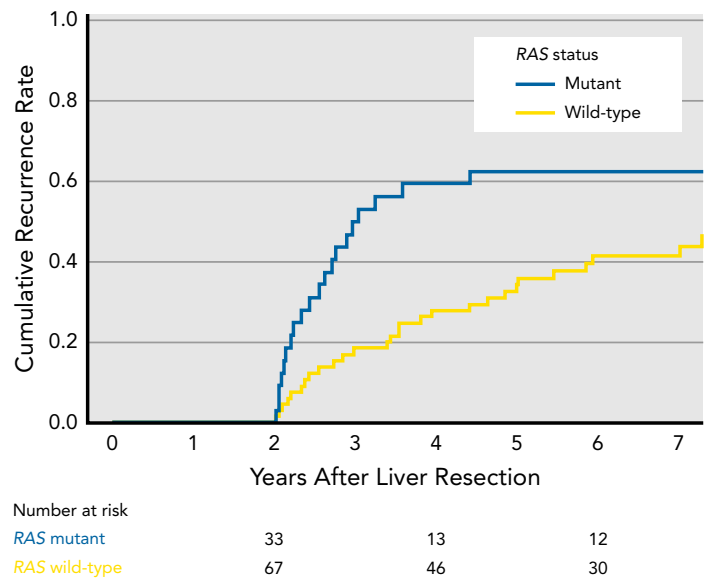

E

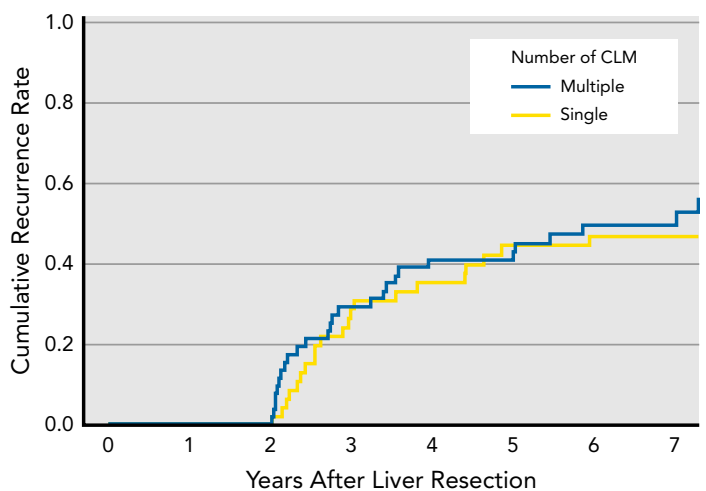

Number at risk

Multiple

52

$\begin{array}{ll}30 & 20 \\ 29 & 22\end{array}$

$\mathbf{F}$

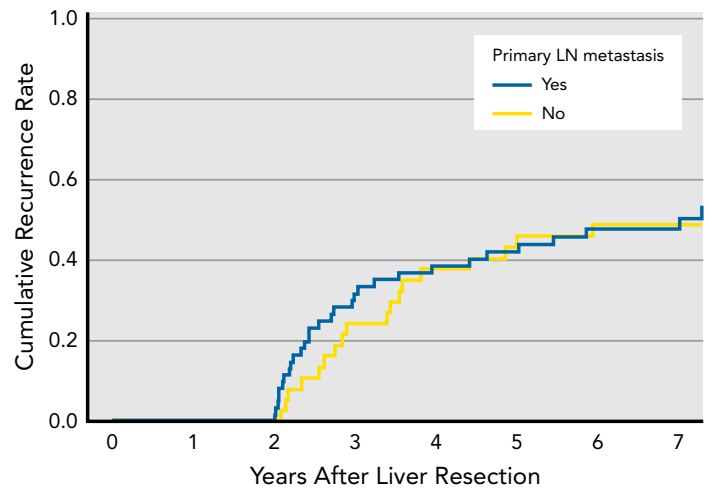

Number at risk

LN metastasis

36
23

25
17

Figure 2. Risk of recurrence over time in the entire cohort by (A) RAS mutation status, (B) number of CLM, and (C) primary LN status. Cumulative recurrence rate by competing risk analysis in patients free from recurrence at 2 years by (D) RAS mutation status, (E) number of CLM, and (F) primary LN status.

Abbreviations: CLM, colorectal liver metastases; LN, lymph node. 
A

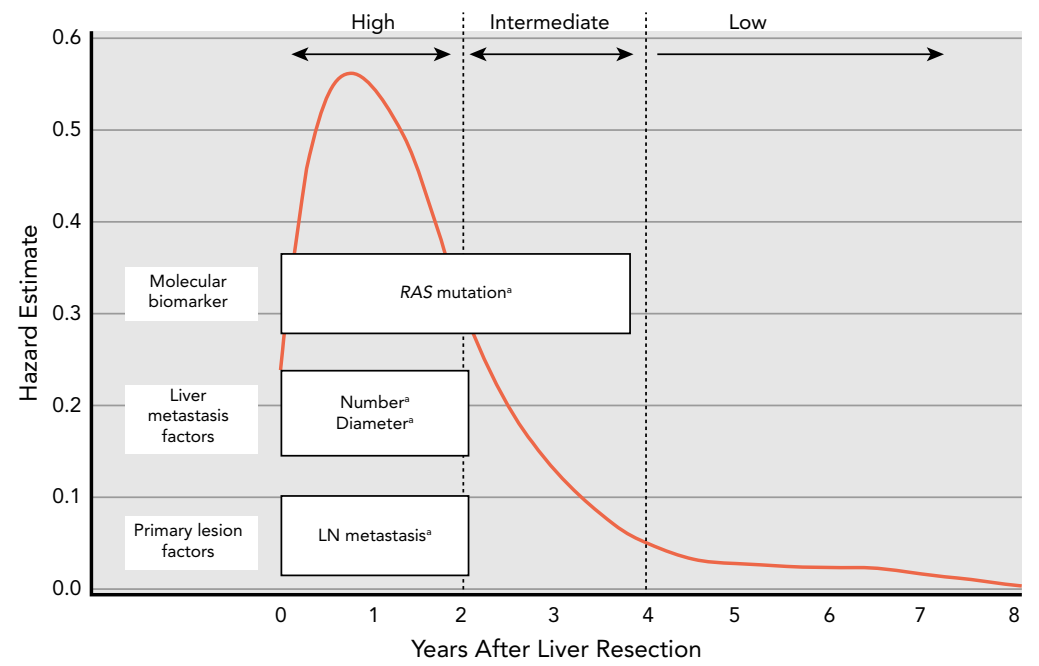

B

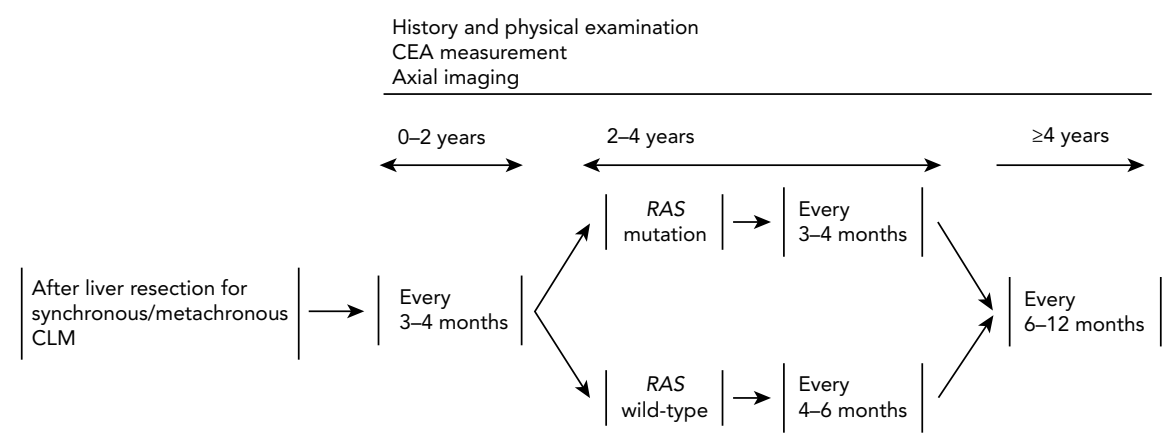

Figure 3. Changes in recurrence risk and risk factors for recurrence over time after CLM resection and a new proposed surveillance algorithm for patients undergoing CLM resection. (A) RAS mutation, multiple CLM, largest liver metastasis diameter $>5 \mathrm{~cm}$, and primary LN metastases were risk factors for recurrence at the time of resection. Only RAS mutation was a risk factor in patients free from recurrence at 2 years. (B) Surveillance algorithm tailored by the changes in recurrence risk and stratified by RAS mutation status. Abbreviations: CLM, colorectal liver metastases; LN, lymph node.

aThe ranges of boxes show the approximate period of risk for recurrence after CLM resection.

that the risk of recurrence at 2 years after CLM resection is approximately 3 times the risk of recurrence at 2 years after resection of primary colorectal tumors, we believe these recommendations are reasonable, especially because there are surgical and nonoperative treatment options for patients with recurrence.

After 2 years without recurrence following CLM resection, we recommend maintaining the surveillance intensity for patients with $R A S$ mutation due to their sustained risk of recurrence, and reducing the surveillance intensity for patients with RAS wild-type. Our surveillance protocol for patients with $R A S$ wild-type 2 to 4 years after CLM resection is similar to or slightly more intensive than other organizations' surveillance recommendations during the first 2 years, which we believe is appropriate given our observation that the risk of recurrence at 4 years after CLM resection in patients recurrence-free at 2 years was similar to the risk of recurrence at 2 years after resection of primary colorectal tumors. After 4 years without recurrence after CLM resection, we further reduce the surveillance intensity because the risk of recurrence decreased to approximately $5 \%$. Thus, our recommendations for surveillance intensity move away from the current one-size-fits-all to a more patient-centered paradigm. Despite the potential benefit of surveillance, it should be noted that testing and clinic visits may increase fear and anxiety in patients and result in a decreasing quality of life. ${ }^{27}$

This study has several potential limitations. First, it is a single-institution retrospective cohort study and covers a long period during which surgical technique and chemotherapy regimens evolved. The recurrence rate was high likely because our institution is a referral academic cancer center with patients with advanced disease. 


\begin{tabular}{|c|c|c|c|}
\hline Parameter & $\begin{array}{l}\text { NCCN } \\
(2020)^{13,14} \\
\text { Stage IV }\end{array}$ & $\begin{array}{l}\text { ASCRS }(2015)^{15} \\
\text { Stages I-III } \\
\text { and IV } \\
\text { IV }\end{array}$ & $\begin{array}{l}\text { ASCO }(2013)^{16} \\
\text { Stages II } \\
\text { and III }\end{array}$ \\
\hline \multicolumn{4}{|c|}{ History and physical examination } \\
\hline $0-2 y$ & Every 3-6 mo & Every 3-6 mo & Every 3-6 mo \\
\hline $2-5 y$ & Every $6 \mathrm{mo}$ & Every $6 \mathrm{mo}$ & Every 3-6 mo \\
\hline \multicolumn{4}{|c|}{ CEA measurement } \\
\hline $0-2 y$ & Every 3-6 mo & Every 3-6 mo & Every 3-6 mo \\
\hline $2-5 y$ & Every 6 mo & Every $6 \mathrm{mo}$ & Every 3-6 mo \\
\hline \multicolumn{4}{|c|}{ Axial imaging } \\
\hline $0-2$ y & Every 3-6 mo & Every $12 \mathrm{mo}$ & Every $12 \mathrm{mo}^{\mathrm{c}}$ \\
\hline $2-5 y$ & Every 6-12 mo & Every $12 \mathrm{mo}$ & Every $12 \mathrm{mo}^{\mathrm{c}, \mathrm{d}}$ \\
\hline
\end{tabular}

Abbreviation: ASCRS, American Society of Colon and Rectal Surgeons.

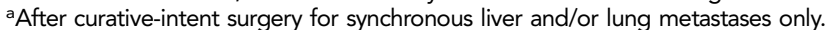

${ }^{b}$ After curative-intent surgery.

'For high-risk patients, every 6-12 months during 0-3 years.

dFor 3 years after surgery.

The observation of recurrence was influenced by our preexisting surveillance protocol. Further, important data were unavailable, including those on socioeconomic, insurance, and marital status; technical resectability of CLM; and treatment approach for synchronous CLM. Follow-up data were missing for $8 \%$ of censored patients because they were not followed at our institution or were lost to follow-up. However, current recommendations are mainly based on expert consensus because no prospectively proved protocol for surveillance after CLM resection has been established. In addition, our long study period allowed us to assess recurrence in a large number of patients with a sufficient follow-up period, which is often missing in surgical studies. The Cox proportional hazards model was based on patients with known RAS mutation status from 2004 to 2011. Therefore, we believe that the bias with respect to period treated and evolution of surgical technique may be minimal. Second, RAS mutation status was known for only $40 \%$ of the cohort analyzed because $R A S$ mutation testing was not performed in patients undergoing CLM resection at our institution before 2003 . Even with this limitation, however, the patient population was large enough for us to perform robust multivariable analyses. Third, we did not assess $B R A F$ mutation status because $B R A F$ is mutated in only $1 \%$ to $6 \%$ of patients, as shown in surgical series. ${ }^{28} \mathrm{~A}$ final limitation is that we did not compare our surveillance strategy with other strategies in terms of whether ours is cost-effective and whether it increases the reintervention rate in patients with asymptomatic recurrences presumably found sooner using cross-sectional imaging.

Despite these limitations, a major strength of this study is that we mitigated the inherent surveillance bias in many studies on this topic by including only patients who had the potential for at least 87 months of follow-up, because the latest observed recurrence in the series occurred at 87 months after CLM resection.

\section{Conclusions}

For patients who undergo liver resection for CLM, the risk of recurrence is highest during the initial 2 years after CLM resection, intermediate 2 to 4 years after CLM resection, and low after 4 years after CLM resection. RAS mutation has a longer-lasting deleterious effect on recurrence than do traditional clinicopathologic risk factors. We therefore suggest that high-surveillance intensity should be maintained for patients with $R A S$ mutation until 4 years after CLM resection. Our algorithm may be helpful for detecting early manifestations of recurrence after CLM resection, which could result in resection with curative intent.

\section{Acknowledgments}

The authors thank Dr. Elena Panettieri for reviewing the data used in the study, Ms. Ruth Haynes for administrative support in the preparation of this article, and Ms. Stephanie Deming for editing the manuscript.

Submitted December 7, 2019; accepted for publication May 21, 2020.

Author contributions: Study concept and design: Kawaguchi, Vauthey. Data acquisition: Kawaguchi, Kopetz, Lillemoe, Tzeng, Chun, Aloia. Data analysis and interpretation: Kawaguchi, Hwang, Wang, Vauthey. Manuscript preparation: Kawaguchi. Critical revision: Kopetz, Lillemoe, Hwang, Wang, Tzeng, Chun, Aloia, Vauthey.

Disclosures: The authors have disclosed that they have not received any financial consideration from any person or organization to support the preparation, analysis, results, or discussion of this article.

Funding: Research reported in this publication was supported by the National Cancer Institute of the National Institutes of Health under award number CA016672.

Disclaimer: The content is solely the responsibility of the authors and does not necessarily represent the official views of the National Institutes of Health

Correspondence: Jean-Nicolas Vauthey, MD, Department of Surgical Oncology, The University of Texas MD Anderson Cancer Center, 1515 Holcombe Boulevard, Unit 1484, Houston, TX 77030. Email: jvauthey@mdanderson.org

\section{References}

1. Manfredi S, Lepage C, Hatem C, et al. Epidemiology and management of liver metastases from colorectal cancer. Ann Surg 2006;244: 254-259.

2. Choti MA, Sitzmann JV, Tiburi MF, et al. Trends in long-term survival following liver resection for hepatic colorectal metastases. Ann Surg 2002; 235:759-766.

3. Abdalla EK, Vauthey JN, Ellis LM, et al. Recurrence and outcomes following hepatic resection, radiofrequency ablation, and combined resection/ablation for colorectal liver metastases. Ann Surg 2004;239: 818-825; discussion 825-827.

4. Fernandez FG, Drebin JA, Linehan DC, et al. Five-year survival after resection of hepatic metastases from colorectal cancer in patients 
screened by positron emission tomography with F-18 fluorodeoxyglucose (FDG-PET). Ann Surg 2004;240:438-447; discussion 447-450.

5. D'Angelica M, Kornprat P, Gonen M, et al. Effect on outcome of recurrence patterns after hepatectomy for colorectal metastases. Ann Surg Oncol 2011;18:1096-1103.

6. Adam R, Bismuth H, Castaing D, et al. Repeat hepatectomy for colorectal liver metastases. Ann Surg 1997;225:51-60; discussion 60-62.

7. Shaw IM, Rees M, Welsh FK, et al. Repeat hepatic resection for recurrent colorectal liver metastases is associated with favourable long-term survival. Br J Surg 2006;93:457-464.

8. Ishiguro S, Akasu T, Fujimoto $Y$, et al. Second hepatectomy for recurrent colorectal liver metastasis: analysis of preoperative prognostic factors. Ann Surg Oncol 2006;13:1579-1587.

9. Battula N, Tsapralis D, Mayer D, et al. Repeat liver resection for recurrent colorectal metastases: a single-centre, 13-year experience. HPB (Oxford) 2014;16:157-163.

10. Saiura A, Yamamoto J, Koga R, et al. Favorable outcome after repeat resection for colorectal liver metastases. Ann Surg Oncol 2014;21: 4293-4299.

11. Gonzalez M, Robert JH, Halkic N, et al. Survival after lung metastasectomy in colorectal cancer patients with previously resected liver metastases. World J Surg 2012;36:386-391.

12. Salah S, Ardissone F, Gonzalez M, et al. Pulmonary metastasectomy in colorectal cancer patients with previously resected liver metastasis: pooled analysis. Ann Surg Oncol 2015;22:1844-1850.

13. Benson $A B$ III, Venook AP, Al-Hawary MM, et al. NCCN Clinical Practice Guidelines in Oncology: Colon Cancer. Version 2.2020. Accessed March 31, 2020. To view the most recent version, visit NCCN.org

14. Benson AB III, Venook AP, Al-Hawary MM, et al. NCCN Clinical Practice Guidelines in Oncology: Rectal Cancer. Version 2.2020. Accessed March 31,2020 . To view the most recent version, visit NCCN.org

15. Steele SR, Chang GJ, Hendren S, et al. Practice guideline for the surveillance of patients after curative treatment of colon and rectal cancer Dis Colon Rectum 2015;58:713-725.

16. Meyerhardt JA, Mangu PB, Flynn PJ, et al. Follow-up care, surveillance protocol, and secondary prevention measures for survivors of colorectal cancer: American Society of Clinical Oncology clinical practice guideline endorsement. J Clin Oncol 2013;31:4465-4470.
17. Gasser TM, Rosenblatt M, eds. Smoothing Techniques for Curve Estimation. Berlin, Germany: Springer; 1979.

18. Gray RJ. A class of $K$-sample tests for comparing the cumulative incidence of a competing risk. Ann Stat 1988;16:1141-1154.

19. Tsikitis VL, Malireddy K, Green EA, et al. Postoperative surveillance recommendations for early stage colon cancer based on results from the clinical outcomes of surgical therapy trial. J Clin Oncol 2009;27: 3671-3676.

20. Wille-Jørgensen P, Syk I, Smedh K, et al. Effect of more vs less frequent follow-up testing on overall and colorectal cancer-specific mortality in patients with stage II or III colorectal cancer: the COLOFOL randomized clinical trial. JAMA 2018;319:2095-2103.

21. Snyder RA, Hu CY, Cuddy A, et al. Association between intensity of posttreatment surveillance testing and detection of recurrence in patients with colorectal cancer. JAMA 2018;319:2104-2115.

22. Rodríguez-Moranta F, Saló J, Arcusa A, et al. Postoperative surveillance in patients with colorectal cancer who have undergone curative resection: a prospective, multicenter, randomized, controlled trial. J Clin Oncol 2006 24:386-393.

23. Primrose JN, Perera R, Gray A, et al. Effect of 3 to 5 years of scheduled CEA and CT follow-up to detect recurrence of colorectal cancer: the FACS randomized clinical trial. JAMA 2014;311:263-270.

24. Moschetti I, Cinquini $M$, Lambertini $M$, et al. Follow-up strategies for women treated for early breast cancer. Cochrane Database Syst Rev 2016; 5:CD001768.

25. Tzeng CW, Fleming JB, Lee JE, et al. Yield of clinical and radiographic surveillance in patients with resected pancreatic adenocarcinoma following multimodal therapy. HPB (Oxford) 2012;14:365-372.

26. Tzeng CW, Abbott DE, Cantor SB, et al. Frequency and intensity of postoperative surveillance after curative treatment of pancreatic cancer: a cost-effectiveness analysis. Ann Surg Oncol 2013;20 2197-2203.

27. Petzel MQ, Parker $\mathrm{NH}$, Valentine $A D$, et al. Fear of cancer recurrence after curative pancreatectomy: a cross-sectional study in survivors of pancreatic and periampullary tumors. Ann Surg Oncol 2012;19: 4078-4084.

28. Kawaguchi Y, Lillemoe HA, Vauthey JN. Gene mutation and surgical technique: suggestion or more? [published online July 18, 2019]. Surg Oncol, doi: 10.1016/j.suronc.2019.07.004 
Supplemental online content for:

\section{A New Surveillance Algorithm After Resection of Colorectal Liver Metastases Based on Changes in Recurrence Risk and RAS Mutation Status}

Yoshikuni Kawaguchi, MD, PhD; Scott Kopetz, MD, PhD; Heather A. Lillemoe, MD; Hyunsoo Hwang, PhD; Xuemei Wang, MS; Ching-Wei D. Tzeng, MD; Yun Shin Chun, MD; Thomas A. Aloia, MD, MHCM; and Jean-Nicolas Vauthey, MD

J Natl Compr Canc Netw 2020;18(11):1500-1508

eFigure 1: Patient Selection

eTable 1: Multivariable HRs of Recurrence-Free Survival After Colorectal Liver Metastases Resection

eTable 2: Multivariable HRs of Recurrence-Free Survival in Patients With RAS Mutation Who Were Recurrence-Free 2 Years After Colorectal Liver Metastases Resection 


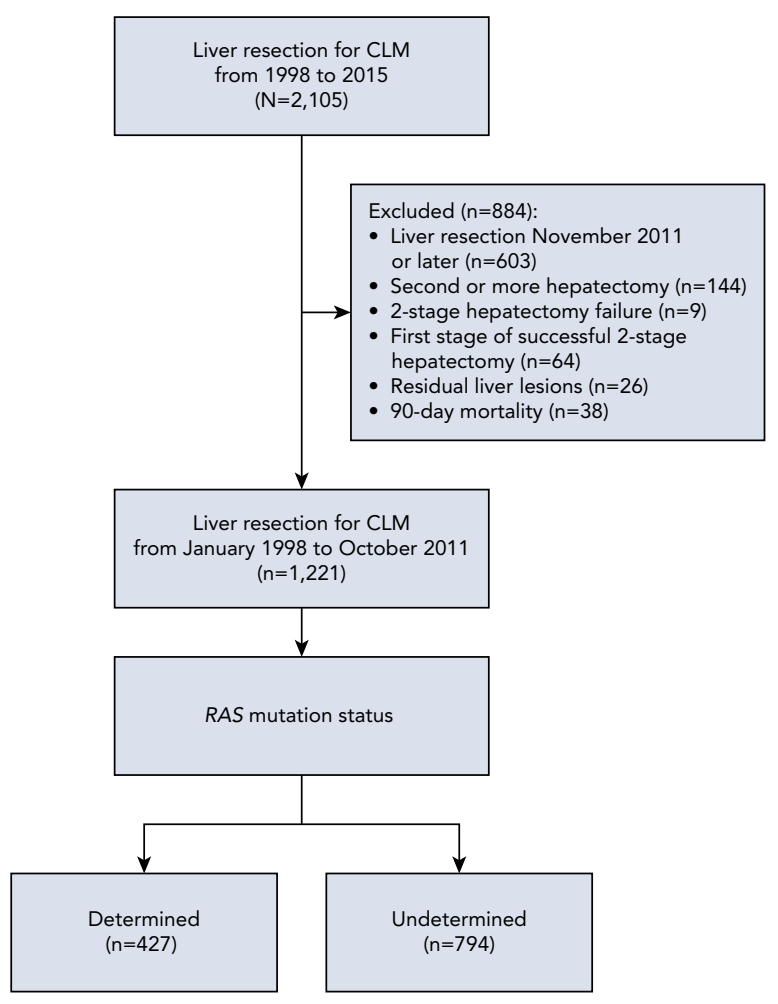

eFigure 1. Patient selection.

Abbreviation: CLM, colorectal liver metastases. 
eTable 1. Multivariable HRs of Recurrence-Free Survival After CLM Resection $(N=1,180)^{a}$

\begin{tabular}{|c|c|c|c|c|c|}
\hline Variable & Patients, n & Events, $\mathrm{n}$ & Multivariable $\mathbf{H R}^{\mathbf{b}}$ & $95 \% \mathrm{Cl}$ & $P$ Value \\
\hline \multicolumn{6}{|l|}{ Sex } \\
\hline Male & 773 & 576 & 1.24 & $1.07-1.43$ & .003 \\
\hline Female & 407 & 244 & Ref & & \\
\hline \multicolumn{6}{|c|}{ Primary lymph node metastases } \\
\hline Yes & 649 & 487 & 1.48 & $1.27-1.72$ & $<.001$ \\
\hline No & 531 & 333 & Ref & & \\
\hline \multicolumn{6}{|c|}{ Number of CLM } \\
\hline Multiple & 649 & 487 & 1.31 & $1.13-1.52$ & $<.001$ \\
\hline Single & 531 & 333 & Ref & & \\
\hline \multicolumn{6}{|c|}{ Largest liver metastasis diameter } \\
\hline$>5 \mathrm{~cm}$ & 211 & 169 & 1.57 & $1.33-1.87$ & $<.001$ \\
\hline$\leq 5 \mathrm{~cm}$ & 969 & 651 & Ref & & \\
\hline \multicolumn{6}{|c|}{ Extrahepatic disease } \\
\hline Yes & 59 & 50 & 1.58 & $1.19-2.11$ & .002 \\
\hline No & 1,121 & 770 & Ref & & \\
\hline \multicolumn{6}{|c|}{ Prehepatectomy chemotherapy } \\
\hline Yes & 899 & 644 & 1.27 & $1.07-1.51$ & .007 \\
\hline No & 281 & 176 & Ref & & \\
\hline \multicolumn{6}{|c|}{ Surgical margin status } \\
\hline R1 & 105 & 92 & 1.63 & $1.31-2.03$ & $<.001$ \\
\hline Ro & 1,075 & 728 & Ref & & \\
\hline
\end{tabular}

Abbreviations: CLM, colorectal liver metastases; HR, hazard ratio.

abased on analysis of data from the 1,180 patients with complete information on T category and lymph node metastasis.

${ }^{b}$ A proportional hazards model was fit for recurrence-free survival. The initial model included the following factors, determined a priori: age (>60 vs $\leq 60$ years), sex primary tumor location, T category, primary lymph node metastasis, prehepatectomy CEA level ( $>200 \mathrm{vs} \leq 200 \mathrm{ng} / \mathrm{mL}$ ), timing of metastasis (synchronous vs metachronous), prehepatectomy chemotherapy, extrahepatic disease, number of CLM (multiple vs single), largest CLM diameter ( $>5 \mathrm{vs} \leq 5 \mathrm{~cm}$ ), surgical margin status (R1 vs R0), and RAS mutation status. Cutoff values of CEA level, number of CLM, and largest CLM diameter were determined according to the Fong score. ${ }^{1}$

\section{Reference}

1. Fong Y, Fortner J, Sun RL, et al. Clinical score for predicting recurrence after hepatic resection for metastatic colorectal cancer: analysis of 1001 consecutive cases. Ann Surg 1999;230:309-318; discussion 318-321. 


\begin{tabular}{|c|c|c|c|c|c|}
\hline \multirow[b]{2}{*}{ Variable } & \multicolumn{5}{|c|}{ RFS } \\
\hline & Patients, $\mathbf{n}$ & Events, $n$ & Multivariable $\mathbf{H R}^{\mathbf{a}}$ & $95 \% \mathrm{Cl}$ & $P$ Value \\
\hline Primary lymph node metastases & 62 & 30 & 1.40 & $0.76-2.58$ & .280 \\
\hline Multiple CLM & 52 & 27 & 1.41 & $0.79-2.52$ & .251 \\
\hline Largest liver metastasis diameter $>5 \mathrm{~cm}$ & 7 & 5 & 1.42 & $0.55-3.64$ & .469 \\
\hline \multicolumn{6}{|l|}{ RAS mutation status } \\
\hline Mutant & 33 & 20 & 2.29 & $1.23-4.26$ & .009 \\
\hline Wild-type & 67 & 28 & Ref & & \\
\hline
\end{tabular}

Abbreviations: CLM, colorectal liver metastases; HR, hazard ratio; RFS, recurrence-free survival.

aA proportional hazards model was fit for RFS. The initial model included the following factors, determined a priori: age ( $>60$ vs $\leq 60$ years), sex, primary tumor location, T category, primary lymph node metastasis, prehepatectomy CEA level ( $>200 \mathrm{vs} \leq 200 \mathrm{ng} / \mathrm{mL}$ ), timing of metastasis (synchronous vs metachronous), prehepatectomy chemotherapy, extrahepatic disease, number of CLM (multiple vs single), largest CLM diameter ( $>5 \mathrm{vs} \leq 5 \mathrm{~cm}$ ), surgical margin status (R1 vs R0), and RAS mutation status. Cutoff values of CEA level, number of CLM, and largest CLM diameter were determined according to the Fong score. ${ }^{1}$

\section{Reference}

1. Fong Y, Fortner J, Sun RL, et al. Clinical score for predicting recurrence after hepatic resection for metastatic colorectal cancer: analysis of 1001 consecutive cases. Ann Surg 1999;230:309-318; discussion 318-321. 